\title{
Seroprevalence of COVID-19 among health workers in the Kathmandu valley, Nepal (SEVID-KaV): a longitudinal cohort study
}

Kiran Raj Pandey ( $\square$ kiran.krp@gmail.com )

Hospital for Advanced Medicine and Surgery

Aseem Bhattarai

Tribhuvan University Teaching Hospital

Suman Pant

Nepal Health Research Council

Rimmy Barakoti

Nepal Health Research Council

Janaki Pandey

Nepal Health Research Council

Anup Subedee

Hospital for Advanced Medicine and Surgery

Prabhat Adhikari

Danphe Care

Diptesh Aryal

Nepal Mediciti Hospital

Prakash Jyoti Pokharel

Civil Service Hospital

Gentle Sunder Shrestha

Tribhuvan University Teaching Hospital

Kamal Pandit

B.P. Koirala Lions Center For Ophthalmic Studies, Tribhuvan University

Narendra Timalsina

Nepal Mediciti Hospital

Sameer Thapa

Nepal Medical College Teaching Hospital

Pradip Gyanwali

Nepal Health Research Council 
Keywords: COVID-19, Seroprevalence, Health workers, Nepal

Posted Date: June 23rd, 2021

DOI: https://doi.org/10.21203/rs.3.rs-626260/v1

License: (c) (1) This work is licensed under a Creative Commons Attribution 4.0 International License. Read Full License 
4

5

22

23

24

25

26
Kiran Raj Pandey, ${ }^{1 *}$ Aseem Bhattarai, ${ }^{2}$ Suman Pant, ${ }^{3}$ Rimmy Barakoti, ${ }^{3}$ Janaki Pandey, ${ }^{3}$ Anup Subedee, ${ }^{1}$ Prabhat Adhikari, ${ }^{4}$ Diptesh Aryal, ${ }^{5}$ Prakash Jyoti Pokharel, ${ }^{6}$ Gentle Sunder Shrestha, ${ }^{2,7}$ Kamal Pandit, ${ }^{8}$ Narendra Timalsina, ${ }^{5}$ Sameer Thapa, ${ }^{9}$ Pradip Gyanwali, ${ }^{3}$

1: Hospital for Advanced Medicine and Surgery, Kathmandu, Nepal

2: Institute of Medicine, Tribhuvan University Teaching Hospital, Kathmandu, Nepal

3: Nepal Health Research Council, Kathmandu, Nepal

4: Danphe Care, Kathmandu, Nepal

5: Nepal Mediciti Hospital, Kathmandu, Nepal

6: Civil Service Hospital, Kathmandu, Nepal

7: Alka Hospital, Kathmandu, Nepal

8: B.P. Koirala Lions Center For Ophthalmic Studies, Institute of Medicine, Tribhuvan University, Kathmandu, Nepal

9: Nepal Medical College Teaching Hospital, Kathmandu, Nepal

*Corresponding author: (KRP)

kiran.krp@gmail.com

Key words: COVID-19, Seroprevalence, Health workers, Nepal 


\section{Abstract}

29 Coronavirus Disease 2019 (COVID-19) burden is often underestimated when relying on case-based incidence

30 reports. Seroprevalence studies accurately estimate infectious disease burden by estimating the population that has 31 developed antibodies following an infection. Sero-Epidemiology of COVID-19 in the Kathmandu valley (SEVID-

$32 \mathrm{KaV}$ ) is a longitudinal survey of hospital-based health workers in the Kathmandu valley. Between December 3-25, 33 we sampled 800 health workers from 20 hospitals and administered a questionnaire eliciting COVID-19 related 34 history and tested for COVID-19 IgG antibodies. We then used a probabilistic multilevel regression model with 35 post-stratification to correct for test accuracy, the effect of hospital-based clustering, and to establish 36 representativeness. 522 (65.2\%) of the participants were female, 372 (46\%) were between ages 18-29, and 7 (0.9\%) 37 were 60 or above. 287 (36\%) of the participants were nurses. About 23\% of the participants previously had a PCR 38 positive infection. 321 (40.13\%) individuals tested positive for COVID-19 antibodies. Adjusted for test accuracy 39 and weighted by age, gender and occupation category, the seroprevalence was 38.17\% (95\% Credible Interval (CrI) 40 29.26\%-47.82\%). Posterior predictive hospital-wise seroprevalence ranged between 38.1\% (95\% CrI 30.7.0\%$41 \quad 44.1 \%)$ and $40.5 \%$ (95\% CrI 34.7\%-47.0\%). 


\section{Introduction}

45

46 Population-based seroprevalence studies have been useful in quantifying the cumulative incidence of the 47 coronavirus disease 2019 (COVID-19) epidemic. Nepal (population 30 million) reported its first infection of SARS-

48 CoV-2 on January 24, 2020-the same week several countries including France, Vietnam, United States, and India 49 reported their first infection. Since then, more than half a million people have been diagnosed with COVID-19 in 50 Nepal, among which more than 7000 have died as of June 2021.(1)

51

52 Nepal's first wave of COVID-19 infections peaked in November 2020. The number of cases fell over the winter, 53 with test positivity rates close to $1 \%$ from a high of about $25 \%$ around October 2020 . Although seroprevalence 54 studies suggest that the cumulative burden of SARS-CoV-2 infection is often several-folds greater than the reported case burden,(2-5) an ongoing second wave that began in March 2021 suggests that a significant percentage of the population was still susceptible to infection after the first wave.

57

Seroprevalence studies are helpful in estimating the true extent of viral spread because they detect seroconversion 59 (i.e. presence of antibodies) after an infection, even among those without clinical or laboratory evidence of active 60 disease. Based on our current understanding, in almost all infected individuals, antibodies against SARS-CoV2 61 appear within 3 weeks of symptom onset.(6,7) When administered to a representative sample of the population, 62 seroprevalence studies can help assess the prevalence of SARS-CoV-2 antibodies as well as cumulative incidence of 63 COVID-19. Seroprevalence studies among at-risk populations like health workers may be a leading indicator of 64 infection burden in the community. In this report, we present results from wave 1 of the Sero-Epidemiology of 65 COVID-19 in the Kathmandu Valley (SEVID-KaV) study, a longitudinal-cohort seroprevalence study among 66 hospital based health workers in Kathmandu, Nepal.

67

\section{Methods}




\section{Study design and population}

71

72 SEVID-KaV study participants were chosen by means of a two-stage cluster-stratified random sampling method. In 73 the first stage, we formed a sampling frame of all hospitals in the Kathmandu valley (population of about 3,000,000) 74 with more than 100 staff-members (63 out of 74 hospitals, with about 25700 staff members), following which we 75 selected 20 hospitals based on the Probability Proportionate to Size (PPS) method.(8) In the second stage, we 76 selected 40 staff members from each of the 20 hospitals based on simple random sampling (SRS) when possible, 77 resulting in a sample size of 800 health workers. This sample size was expected to estimate the seroprevalence with 78 a margin of error of less than 5\%. PPS with SRS ensures that each health worker in the selected hospitals has the 79 same probability of being sampled in the study.(8)

80

81 Sampling was conducted between December 3 to 25, 2020. Hospital staff (clinical as well as administrative) above 8218 years of age were eligible to participate. Staff names were obtained from hospital human resources departments 83 and randomly ordered using a spread-sheet. Hospital staff were then telephoned in that random order until 40 84 participants could be recruited for the study. Six hospitals were unable to provide phone numbers for staff members. 85 There, a convenience sample of 40 staff members was randomly sampled from among those present at the hospital on the day of sampling.

Ethical approval for all study sites was granted by the Nepal Health Research Council's Ethical Review Board (ERB) (Approval reference number: 609). All except two hospitals that were selected granted written permission to conduct the study at their site. The two hospitals that were not able to grant permission in time were replaced with

91 hospitals that were close by and had a similar staff-size.

93 Study enumerators spent 1-2 days at each study site where they administered a study questionnaire to the 40 pre94 selected participants after obtaining a written informed consent. The study questionnaire elicited information on 95 demographics, symptoms, testing, contacts and travel history. Enumerators also obtained 4-5 ml of blood samples 96 from each participant. Blood samples were then transported to the Biochemistry Labs at Tribhuvan University 97 Teaching Hospital (TUTH), and tested for antibodies against SARS-CoV-2. 


\section{Antibody test and validation}

100

101

A Chemiluminescence Immunoassay (CLIA) based antibody test from Ortho Clinical Diagnostics (Vitros CoV2G

102

[IgG]) was used to assess for the presence of antibodies to establish past exposure to SARS-CoV-2 among study

individuals.(9) The test detects IgG antibodies against S1 spike protein of SARS-CoV-2. We performed a local validation of the antibody test with 77 positive controls and 65 negative controls. Positive controls were serum samples from individuals with a positive polymerase chain reaction (PCR) test more than three weeks prior and negative controls were frozen serum samples that were obtained before December 2019. Manufacturer recommended test sample:calibrator optical signal ratio of 1.0 or more was used to identify positive results. We calculated the sensitivity and specificity of the serology test by fitting a Beta-Binomial Bayesian model using data from our validation study. We also used validation data submitted by the test manufacturer to regulatory agencies to generate strongly informative priors for the Beta-Binomial model.(10) Based on the data, the sensitivity and specificity of the test in our context were 89.3\% (95\% Credible Interval (CrI), 85.8-93.0) and 99.2\% (95\% CrI 98.1\%-99.8\%) respectively.

\section{Statistical analysis}

The statistical analysis aimed to account for test accuracy in calculating the population based seroprevalence, to

117 account for the effects of hospital based clustering, and to make the study findings representative of the study

118 population. To do the first, we modeled the serology test result as a Bernoulli process. We then used Bayes' rule to 119 account for the test inaccuracy by populating it with measures of test sensitivity and specificity. To account for 120 clustering at the hospital, we extended this model to a hierarchical Bayesian logistic regression model with partial 121 pooling. To ensure representativeness of the study, we further extended this model into a multilevel (or hierarchical) 122 regression model with post-stratification (MRP) by including age, gender, and occupation as predictors. We then 
124 seroprevalence among health workers in Kathmandu. The statistical framework for our analysis is represented as 125 below:(2,11-15)

126 $\mathrm{x}_{\mathrm{i}} \sim$ Bernoulli $\left(\mathrm{p}_{\mathrm{i}}^{*}\right.$ sens $\left.+\left(1-\mathrm{p}_{\mathrm{i}}\right) *(1-\mathrm{spec})\right)$

127

$p_{i}=\operatorname{logit}^{-1}\left(a l\right.$ pha $\left.+\alpha_{h}{ }^{*} \operatorname{sigma}+x_{i} \beta\right)$

128

$p \sim \operatorname{Normal}(0.2,1)$

129

alpha $\sim \operatorname{Normal}(0,1)$

130

$\alpha_{h} \sim \operatorname{Normal}(0,1)$

131

sens Beta $(71,9)$

132

spec $\sim \operatorname{Beta}(440,2)$

133

$\mathrm{x}^{+} \sim \operatorname{Binomial}\left(\mathrm{n}^{+}\right.$, sens $)$

134

$\left.\mathrm{x}^{-} \sim \operatorname{Binomial}\left(\mathrm{n}^{-}, \mathrm{spec}\right)\right)$

135

136

$\mathrm{p}\left(\mathrm{y}_{\text {pred }} \mid \mathrm{y}\right) \sim \int_{0}^{1} \mathrm{p}\left(\mathrm{y}_{\text {pred }} \mid\right.$ theta $){ }^{*} \mathrm{p}($ theta $\mid \mathrm{y}) \mathrm{d}($ theta $)$

137

138

Relative Risk (RR) for group g:

139

$\left(R_{g}\right)=p_{g} / p=\operatorname{logit}^{-1}\left(\right.$ alpha $\left.+\beta_{0}+\beta_{g}+\alpha_{h}{ }^{*} \operatorname{sigma}\right) / \operatorname{logit}{ }^{-1}\left(a l p h a+\beta_{0}+\alpha_{h}{ }^{*} s_{g m a}\right)$

140 Here, $x_{i}$ is the result of the sero-survey for the $i^{\text {th }}$ individuals, $p_{i}$ is the true underlying probability of a positive test

141 for the $i^{\text {th }}$ individual, sens is the test sensitivity, spec is the test specificity, alpha is the fixed intercept term,

142 sigma is the standard deviation for the hospital random effect and, $\alpha_{h}$ is the extent of deviation of the random

143 effect in terms of sigma. $X_{i}$ is a vector of predictor variables (age group, gender and occupation) and $\beta$ is a vector of

144 their respective coefficients. Theta represents the fitted parameters, $y_{\text {pred }}$ is the new predicted data. Based on 145 previous findings, we assumed a weakly informative normally distributed prior for the overall seroprevalence with a 
146

147

148

149

150

151

152

153

154

155

156

157

158

159

160

161

\section{Results}

163

164

165

166

167

168

169

170

171

172

173 appendix.

mean of 0.2 and a standard deviation of 1 . We created 40 strata (4 age categories x 2 gender categories $\times 5$ occupation categories), and calculated seroprevalence for each of these 40 strata, which we then multiplied by their respective population weights to obtain the final seroprevalence.

We implemented this probabilistic model in the Stan programming language and interfaced it in R (version 4.0.3), via the Rstan package.(16,17) Stan samples the posterior parameter space using Hamiltonian Monte Carlo (HMC) No U-Turn Sampler (NUTS). We ran 4 chains with 5000 iterations per chain and discarded the first 1000, resulting in 16,000 sampling iterations. To assess for model convergence, we used the $\mathrm{R}$ hat statistic, the number of effective samples, the energy parameter and visual measures. Visual model diagnostics are given in the supplementary

Overall and hospital-wise seroprevalence among health workers is reported as the mean and the 95\% Credible Interval (CI) of the conditional probability of seropositivity given the data. Effect sizes are reported in terms of odds or relative risks. While calculating relative risk, the largest groups (age group 18-29, female gender, and nurses) were considered the respective reference groups.

821 participants from 20 clusters (hospitals) participated in the survey. For each of the 20 clusters, we included the first 40 participants based on their order in the randomized sampling list, resulting in 800 records in our analytic sample. Of these 800 individuals, 522 (65.2\%) were female, 372 (46\%) were between ages 18-29, and 7 (0.9\%) were 60 or above. 287 (36\%) of the participants were nurses, 172 (22\%) were administrative staff, 147 (18\%) were doctors, 56 (7\%) were laboratory and pharmacy staff while 137 (17\%) were other staff with clinical or bedside roles. 529 (66\%) were married, 485 (61\%) had a bachelor’s degree or higher while 6\% had no formal education (Table 1). In comparison, based on records at the health ministry, $63 \%$ of health workers in the Kathmandu valley are females, $42 \%$ are between ages $18-29,3 \%$ are above age $60,30 \%$ are nurses, $36 \%$ are administrative staff and $20 \%$ are doctors. Additional details are available in the supplementary appendix. 
174 Table 1: Demographic characteristics of SEVID-KaV participants based on SARS-CoV-2 antibody status

\begin{tabular}{|c|c|c|c|c|}
\hline \multirow[b]{2}{*}{ Characteristic } & \multicolumn{4}{|c|}{ Antibody Status } \\
\hline & $\begin{array}{c}\text { Overall } \\
(\mathrm{N}=800)^{1}\end{array}$ & $\begin{array}{l}\text { Negative } \\
(\mathrm{N}=479)^{1}\end{array}$ & $\begin{array}{l}\text { Positive } \\
(\mathrm{N}=321)\end{array}$ & $\begin{array}{c}\mathrm{P}- \\
\text { value }^{2}\end{array}$ \\
\hline Age Group & & & & 0.2 \\
\hline $18-29$ & 372 ) & $214(58 \%)$ & $158(42 \%)$ & \\
\hline $30-49$ & 355 & $213(60 \%)$ & $142(40 \%)$ & \\
\hline $50-59$ & 66 & $47(71 \%)$ & $19(29 \%)$ & \\
\hline$>=60$ & 7 & $5(71 \%)$ & $2(29 \%)$ & \\
\hline Gender & & & & 0.3 \\
\hline Female & 522 & $305(58 \%)$ & $217(42 \%)$ & \\
\hline Male & $278 \%)$ & $174(63 \%)$ & $104(37 \%)$ & \\
\hline Occupation & & & & 0.8 \\
\hline Nurse & 288 & $167(58 \%)$ & $121(42 \%)$ & \\
\hline Doctor & 147 & $90(61 \%)$ & $57(39 \%)$ & \\
\hline $\begin{array}{l}\text { Other bedside/ patient-care } \\
\text { role (e.g. patient transport) }\end{array}$ & 137 & $79(58 \%)$ & $58(42 \%)$ & \\
\hline Laboratory/ Pharmacy & 56 & $34(61 \%)$ & $22(39 \%)$ & \\
\hline $\begin{array}{l}\text { Administration (including } \\
\text { security) }\end{array}$ & 172 & $109(63 \%)$ & $63(37 \%)$ & \\
\hline Marital Status & & & & 0.2 \\
\hline Married & 529 & $319(60 \%)$ & $210(40 \%)$ & \\
\hline Unmarried & 266 & $155(58 \%)$ & $111(42 \%)$ & \\
\hline $\begin{array}{l}\text { Divorced /Separated/ } \\
\text { Widowed }\end{array}$ & 5 & $5(100 \%)$ & $0(0 \%)$ & \\
\hline Education & & & & 0.074 \\
\hline Illiterate & 25 & $9(36 \%)$ & $16(64 \%)$ & \\
\hline $\begin{array}{l}\text { Literate but no formal } \\
\text { education }\end{array}$ & 23 & $11(48 \%)$ & $12(52 \%)$ & \\
\hline $\begin{array}{l}\text { Primary education (Grade } 5 \\
\text { or below) }\end{array}$ & 26 & $14(54 \%)$ & $12(46 \%)$ & \\
\hline $\begin{array}{l}\text { Secondary education } \\
\text { (Grade } 6 \text { to } 12 \text { ) }\end{array}$ & 241 & $145(60 \%)$ & $96(40 \%)$ & \\
\hline Bachelor degree or higher & 485 & $300(62 \%)$ & $185(38 \%)$ & \\
\hline Monthly Income & & & & 0.6 \\
\hline Up to Rs 20,000 & 131 & $74(56 \%)$ & $57(44 \%)$ & \\
\hline
\end{tabular}




$\begin{array}{lccc}\text { Rs 20,001-50,000 } & 271 & 156(58 \%) & 115(42 \%) \\ \text { Rs 50,001-100,000 } & 164 & 103(63 \%) & 61(37 \%) \\ \text { More than 100,000 } & 136 & 86(63 \%) & 50(37 \%) \\ \text { Don't know/ can't say } & 98 & 60(61 \%) & 38(39 \%)\end{array}$

in (\%) 2Fisher's exact test; Pearson's Chi-squared test

Percentages are in terms of the row total. One participant had missing occupation data and was coded as a Nurse (the largest group). 1 US $\$=\sim 117$ Rs.

177 Table 2 presents the frequency of common COVID-19 symptoms since January 2020 among seropositive and seronegative individuals. The symptoms with the unadjusted odds of seropositivity greater than one (i.e. more specific) were a loss of smell (odds of 4.64), fever (3), shortness of breath 2.35), muscle ache, diarrhea, rash and joint pain. The unadjusted odds of seropositivity among individuals with cough were 0.95 and headache were 0.94 .

182 Figure 1: Unadjusted seroprevalence of COVID-19 among health workers in the Kathmandu 183 valley

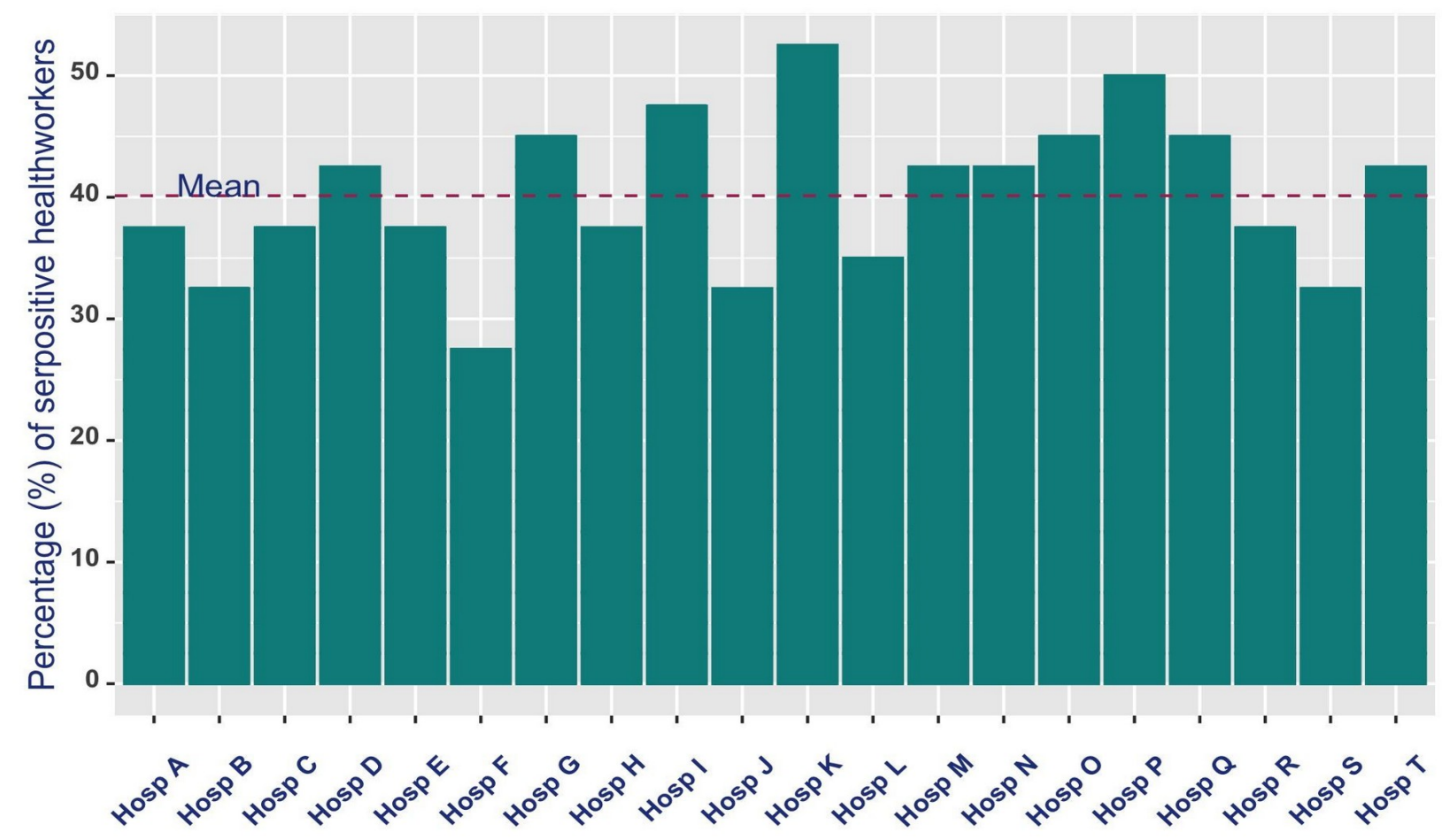

Note: Dotted line represents the mean unadjusted overall seroprevalence. Cyan bars represent unadjusted seroprevalence for 
187

188 Only about $70 \%$ of seropositive individuals in our study had one or more of the specific symptoms. Even when

189 cough (with odds of seropositivity less than 1), was included among the symptoms, only 75\% of the seropositive

190 individuals had at least one symptom. Although 597 of the 800 participants had at least one PCR test. 181

191 participants (i.e. 23\%) had a positive PCR test in the past. Odds of seroconversion among health workers who had a

192 positive test in the past were 3.02, while they were 0.34 among individuals who had a negative PCR . Among health

193 workers who did not have a PCR test in the past, the odds of sero-conversion were 0.60 (corresponding to a

194 probability of $37 \%)$.

195

196 Table 2: COVID-19 related symptoms in SEVID-KaV study participants

\begin{tabular}{|c|c|c|c|c|}
\hline \multirow[b]{2}{*}{ Symptom } & \multicolumn{4}{|c|}{ Antibody Status } \\
\hline & $\begin{array}{c}\text { Overall } \\
(\mathrm{N}=800)^{1}\end{array}$ & $\begin{array}{l}\text { Negative } \\
(\mathrm{N}=479)^{1}\end{array}$ & $\begin{array}{l}\text { Positive } \\
(\mathrm{N}=321)^{1}\end{array}$ & $p$-value ${ }^{2}$ \\
\hline Fever & 172 & $43(25 \%)$ & $129(75 \%)$ & $<0.001$ \\
\hline $\begin{array}{l}\text { Shortness of } \\
\text { Breath }\end{array}$ & 94 & $28(30 \%)$ & $66(70 \%)$ & $<0.001$ \\
\hline Cough & 277 & $140(51 \%)$ & $137(49 \%)$ & $<0.001$ \\
\hline $\begin{array}{l}\text { Sputum } \\
\text { Production }\end{array}$ & 77 & $42(55 \%)$ & $35(45 \%)$ & 0.3 \\
\hline Loss of Smell & 141 & $25(18 \%)$ & $116(82 \%)$ & $<0.001$ \\
\hline Headache & 372 & $192(52 \%)$ & $180(48 \%)$ & $<0.001$ \\
\hline Myalgia & 257 & $104(40 \%)$ & $153(60 \%)$ & $<0.001$ \\
\hline Diarrhoea & 79 & $33(42 \%)$ & $46(58 \%)$ & $<0.001$ \\
\hline Rash & 25 & $9(36 \%)$ & $16(64 \%)$ & 0.013 \\
\hline Joint Pain & 115 & $49(43 \%)$ & $66(57 \%)$ & $<0.001$ \\
\hline
\end{tabular}

in (\%) 2Pearson's Chi-squared test

Percentages are in terms of the row total. Columns add up to more than the column total because many individuals reported more than one symptom. Any report of symptoms since January 2020, when the pandemic started, is recorded as a positive.

199321 of the 800 health workers included in the analysis tested positive for COVID-19 antibodies. A pooled (nonhierarchical) model of the overall seroprevalence without adjusting for test accuracy (sensitivity and specificity) 
201 resulted in an unadjusted seroprevalence of 40.2\% (95\% CrI 36.8-43.6\%). A model with no pooling between

202 hospitals, unadjusted for test accuracy resulted in hospital-wise seroprevalence that ranged between $28.61 \%$ (95\%

203 CrI 16.17\%-43.08\%) and 52.15\% (95\% CrI 37.40\%-67.01\%) (Figure 1). Adjusted for test accuracy, the

204 seroprevalence estimate from the unpooled model ranged between 33.6\% (95\% CrI 18.5\%-51.5\%) to 62.2\% (95\%

205 CrI 44.0\%-80.7\%). These results are presented in Table 2.

206

207 Table 3: Relative risk of COVID-19 seropositivity based on age, gender and health worker

208 occupation

\begin{tabular}{|c|c|c|}
\hline & $\begin{array}{c}\text { Mean } \\
\text { Seroprevale } \\
\text { nce \% }\end{array}$ & $\begin{array}{c}\text { Relative Risk } \\
\text { (95\% Credible } \\
\text { Interval) }\end{array}$ \\
\hline \multicolumn{3}{|l|}{ Age Group } \\
\hline $18-29^{\#}$ & 41.7 & 1 \\
\hline $30-49$ & 40.5 & $0.97(0.81-1.2)$ \\
\hline $50-59$ & 30.7 & $0.74(0.48-1)$ \\
\hline $60<=$ & 36.6 & $0.88(0.31-1.6)$ \\
\hline \multicolumn{3}{|l|}{ Gender } \\
\hline Female $^{\#}$ & 42.0 & 1 \\
\hline Male & 39.0 & $0.94(0.75-1.1)$ \\
\hline \multicolumn{3}{|l|}{ Occupation } \\
\hline Nurse $^{\#}$ & 41.7 & 1 \\
\hline Administration & 39.0 & $0.94(0.72-1.2)$ \\
\hline Bedside support & 44.3 & $1.1(0.83-1.3)$ \\
\hline Doctor & 40.8 & $0.98(0.74-1.3)$ \\
\hline $\begin{array}{l}\text { Laboratory/ } \\
\text { Pharmacy } \\
\text { Personnel }\end{array}$ & 41.8 & $0.99(0.69-1.3)$ \\
\hline
\end{tabular}

\#Reference group. Administration includes personnel that do not have a direct patient care responsibility including security personnel. Beside support refers to nurses aides and patient transporters. 
Hospital-wise seroprevalence calculated from the fitted parameters generated from the final multilevel model with

211

212

213

214

215

216

217

218

219

post-stratification was between 38.1\% (95\% CrI 30.7\%-44.1\%) and 40.5\% (95\% CrI 34.7\%-47.0\%) (Figure 2).

Overall seroprevalence based on the final multilevel model with post-stratification, adjusted for test sensitivity and specificity was 38.99\% (95\% CrI 29.08\%-43.91\%). When weighted based on the age group, gender and occupation

of health workers in the Kathmandu valley, the seroprevalence was 38.17\% (95\% CrI 29.26\%-47.82\%). Relative risk of seropositivity was the greatest among 18-29 year olds, females and bedside care providers, however many of these differences did not achieve statistical significance at the 95\% credible interval (Table 3).

Figure 2: Adjusted hospital-wise seroprevalence of COVID-19 among health workers in the Kathmandu valley

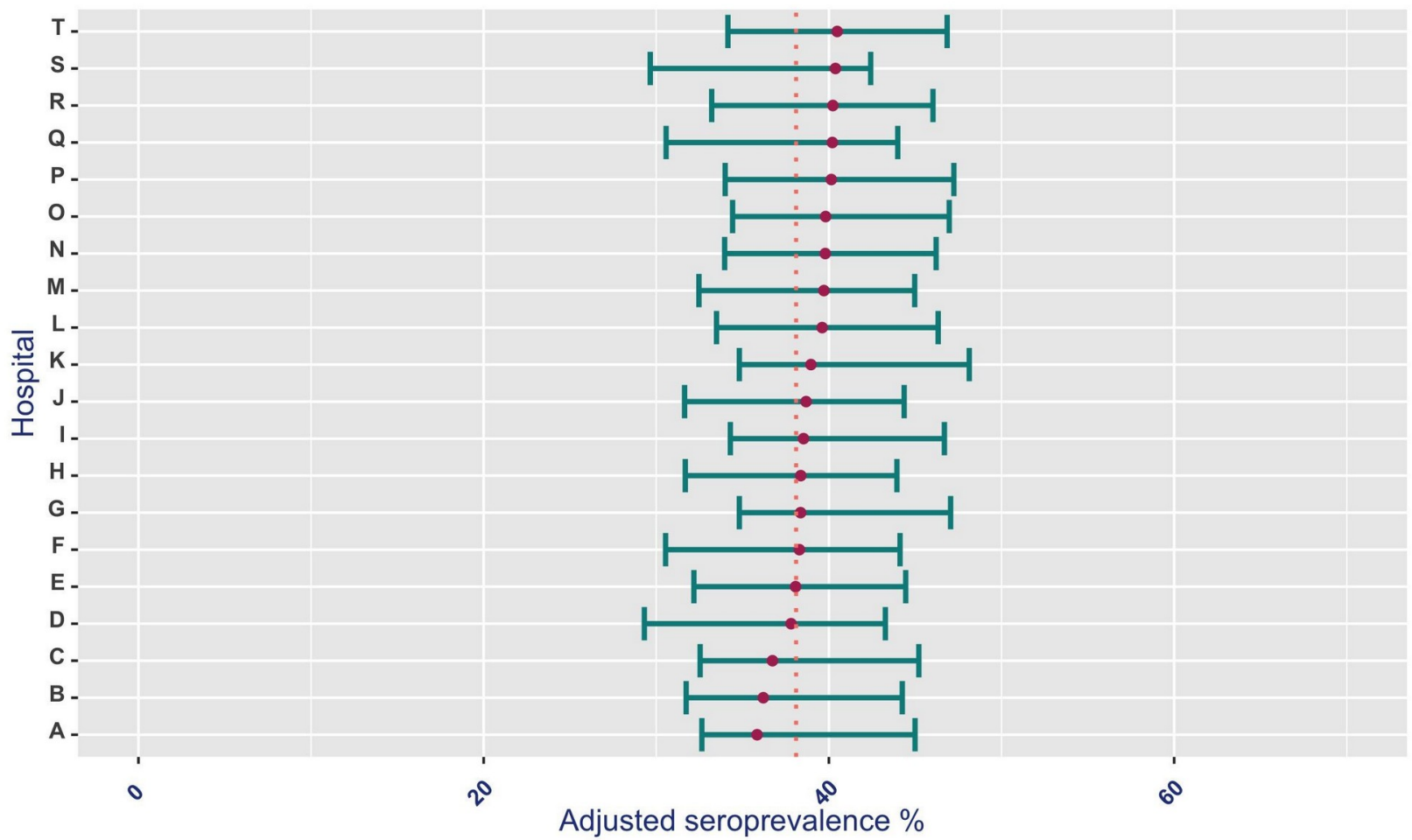

Note: Red dots represent the mean seroprevalence for each hospital. Cyan error bars represent the 95\% central predictive interval of the seroprevalence for each hospital. The dotted line represents the overall adjusted seroprevalence. 


\section{Discussion}

228

229 Our analysis of the prevalence of antibodies against SARS-CoV-2 among hospital-based health workers in the

230 Kathmandu valley, Nepal reveals a substantial exposure to the infection by the winter of 2020. By mid-December

231 2020, about $40 \%$ of the health workers had already developed antibodies against SARS-CoV-2, well before

232 vaccination against COVID-19 had begun in Nepal. This means that these health workers had already been exposed

233 to SARS-CoV-2 by the end of November, 2020 and subsequently developed antibodies against it. In addition, our

234 findings show that seroprevalence is marginally higher (trending toward statistical significance) among health

235 workers who have a direct patient care role (nursing and bedside clinical support roles). COVID-19 seroprevalence

236 also appears to be inversely correlated—albeit weakly—with socioeconomic indicators (educational attainment and

237 financial status). Although a large proportion of seropositive health workers experienced at least one symptom that

238 was consistent with COVID-19, the most specific symptoms were a loss of sense of taste or smell, fever and

239 shortness of breath. As expected, a positive PCR test in the past increased the odds of seropositivity substantially.

240

241 Because of the nature of their work, health care workers were thought to have a potentially higher risk of exposure

242 to SARS-CoV-2. There are reasons for such expectations. Early in the epidemic, several countries, including Nepal,

243 faced a shortage of personal protective equipment. This might have led to greater workplace exposure to SARS-

244 CoV-2 among health workers. In addition, early in the epidemic there was also an inadequate understanding of the

245 risk and mode of transmission (for e.g. transmission from asymptomatic individuals and airborne transmission or

246 higher risk of transmission in unventilated closed spaces), this could have resulted in high risk of infection among

247 health and other frontline workers.

248

249 Although the infection burden among health workers as shown by our study is substantial, it is not clear the extent to

250 which this burden is different from the disease burden at the level of the community. Several studies have shown

251 that seroprevalence among health workers often tracks seroprevalence in the community.(18-22) And contrary to

252 earlier expectations, at least a few studies have shown that in hospitals where adequate infection control practices

253 are in place, health workers have a low risk of contracting the infection in the workplace. $(19,23)$ Therefore, it is

254 unclear whether the seroprevalence seen in our study, although substantial, is the consequence of risk of exposure at 
255

256

257 258

259

260

261

262

263

264

265

266

267

268

269

270

271

272 Our study has several strengths. First, the study was designed to be representative of all the hospital based health

the workplace or the high community burden of the infection in Kathmandu. Across densely populated urban communities of South Asia, there appears to have been a significant spread of COVID-19 within the first year of the pandemic. An as yet unpublished estimate indicates that at least $17 \%$ of the overall population in and around Kathmandu may have already been infected by September 2020.(24) In pockets of urban India, where COVID-19 related epidemic dynamics are similar in many ways to Kathmandu, this proportion was found to be even higher. $(25-27)$

The 38\% seroprevalence among the valley's 25,000 or so health workers indicates that about 10,000 had contracted the infection by the end of November, however official reports indicate that until then, only about 2500 of them had been diagnosed.(28) This is even while health workers have comparatively better access to testing —many facilities in Kathmandu routinely test their staff on a periodic basis. In fact, about $80 \%$ of the health workers in our sample had already had at least one PCR test as part of routine surveillance. The four fold gap between cases and infections is partly explained by the fact many individuals who contracted the infection appear to have developed no symptoms at all, or experienced mild symptoms for which they did not seek testing. In the general population, the gap between cases and infections could be even higher as they have poorer access to testing services, or may not seek care in the first place.

273 workers in the Kathmandu valley. As our comparison with the overall health worker population of Kathmandu 274 shows, our sampled population appears to well represent Kathmandu's health workers based on their age group, 275 gender and occupational group. In addition, our study post-stratified and weighted seroprevalence based on these 276 demographic variables to make the findings representative of the study population. The fact that post-stratification 277 resulted in less than a percentage difference between the unweighted and weighted seroprevalence means that our 278 study sample was remarkably representative of the overall health worker population. Our methodology allowed for 279 an easy correction of test accuracy. The study sampled more than $3 \%$ of the study population. The comparatively 280 large sample size, combined with the hierarchical statistical framework allowed for more accurate estimates of 281 seroprevalence. The longitudinal cohort design of this study means that it will allow us to study not only the 282 temporal variation of seroprevalence but also to study antibody decay in the future. 
284 A major limitation of this study is our measure of test accuracy. Our unadjusted measure for sensitivity was $82 \%$

285 while it was $90 \%$ based on the manufacturer's data alone. It is possible that some of the PCR test results that we 286 used to identify positive controls were falsely positive, especially among individuals who did not have a clinical

287

288

289

290

291

292

293

294

295

296

297

\section{Conclusions}

299

300

301

302

303

304

305

306

307

308 309 balanced. diagnosis of COVID-19. To account for this limitation, we derived strongly informative Bayesian priors from the manufacturer's data and allowed them to influence our final calculation of test accuracy. Because of this, our measure of the test accuracy in the final model-with an $89 \%$ sensitivity and greater than $99 \%$ specificity closely matches manufacturer's data. The added benefit of our validation data may be that it may reflect upon local testing conditions. Surveys like these might be biased because individuals who agree to participate in such surveys may have a greater tendency to seek care, or may be at a higher risk. In our survey, $40 \%$ of those invited agreed to participate and an overwhelming majority of those who declined cited scheduling conflict as the reason for not participating. In addition, unadjusted seroprevalence among those who had not had a PCR test in the past (correlating to health care seeking behaviour and risk), was 37\%, meaning that our survey sample was fairly well

A significant proportion of health workers in Kathmandu appear to have been infected with COVID-19 by the end of 2020. Although it is not entirely clear to what extent health workers were infected at the workplace, these seroprevalence figures still warrant a reassessment of infection control practices at Kathmandu's hospitals. If health worker and community seroprevalence are correlated, these estimates may also be indicative of the seroprevalence in the overall community. Since seroprevalence studies are easier to conduct among health workers than in the community, they could be used to inform the serostatus of the overall community and predict future epidemic dynamics and disease spread. Future waves of this study will be useful in assessing the progress of the epidemic over time, the temporal variation of antibodies, and now that a sizable proportion of health workers have been vaccinated, they could also generate insight on the real world evidence of vaccine efficacy. 
Bibliography

312 1. Ministry of Health and Population. COVID19-Dashboard [Internet]. [cited 2021 Feb 22].

313 Available from: https://covid19.mohp.gov.np/

2. Stringhini S, Wisniak A, Piumatti G, Azman AS, Lauer SA, Baysson $\mathrm{H}$, et al.

316

8. Skinner CJ. Probability proportional to size (PPS) sampling. In: Balakrishnan N, Colton T, Everitt B, Piegorsch W, Ruggeri F, Teugels JL, editors. Wiley statsref: statistics reference online. Chichester, UK: John Wiley \& Sons, Ltd; 2014.

9. Ortho Clinical Diagnostics. Ortho Clinical Diagnostics antibody tests [Internet]. 2020 [cited 2021 Feb 26]. Available from:

https://www.orthoclinicaldiagnostics.com/global/covid19/antibody-test

10. Food and Drug Administration (FDA). EUA Authorized Serology Test Performance [Internet]. 2020 [cited 2021 Jan 10]. Available from:

https://www.fda.gov/medical-devices/coronavirus-disease-2019-covid-19-emergency-useauthorizations-medical-devices/eua-authorized-serology-test-performance

11. Makela S, Si Y, Gelman A. Bayesian inference under cluster sampling with probability proportional to size. Stat Med. 2018 Nov 20;37(26):3849-68.

12. Gelman A, Carpenter B. Bayesian analysis of tests with unknown specificity and sensitivity. J Royal Statistical Soc C. 2020 Aug 13;

13. Carpenter B. Hierarchical Partial Pooling for Repeated Binary Trials [Internet]. 2016 [cited 2021 May 19]. Available from: https://mc-stan.org/users/documentation/case-studies/poolbinary-trials.html

14. Stan Development Team. Hierarchical logistic regression [Internet]. Stan User's Guide 
350

351

352

353

354

355

356

357

358

359

360

361

362

363

364

365

366

367

368

369

370

371

372

373

374

375

376

377

378

379

380

381

382

383

384

385

386

387

388
2.26. 2021 [cited 2021 May 20]. Available from: https://mc-stan.org/docs/2_26/stan-usersguide/hierarchical-logistic-regression.html

15. Stan Development Team. Multilevel regression and poststratification [Internet]. Stan User's Guide 2.26. 2021 [cited 2021 Jun 3]. Available from: https://mc-stan.org/docs/2_23/stan-users-guide/multilevel-regression-andpoststratification.html

16. Stan Development Team. RStan: the R interface to Stan [Internet]. Stan Development Team; 2020 [cited $2021 \mathrm{Feb} 26$ ]. Available from: https://mc-stan.org

17. Carpenter B, Gelman A, Hoffman MD, Lee D, Goodrich B, Betancourt M, et al. stan: A probabilistic programming language. J Stat Softw. 2017;76(1):1-32.

18. Iruretagoyena M, Vial MR, Spencer-Sandino M, Gaete P, Peters A, Delgado I, et al. Longitudinal assessment of SARS-CoV-2 IgG seroconversion among front-line healthcare workers during the first wave of the Covid-19 pandemic at a tertiary-care hospital in Chile. BMC Infect Dis. 2021 May 26;21(1):478.

19. Fukuda H, Seyama K, Ito K, Ai T, Nojiri S, Hori S, et al. SARS-CoV-2 seroprevalence in healthcare workers at a frontline hospital in Tokyo. Sci Rep. 2021 Apr 16;11(1):8380.

20. Rodriguez A, Arrizabalaga-Asenjo M, Fernandez-Baca V, Lainez MP, Al Nakeeb Z, Garcia JD, et al. Seroprevalence of SARS-CoV-2 antibody among healthcare workers in a university hospital in Mallorca, Spain, during the first wave of the COVID-19 pandemic. Int J Infect Dis. 2021 Apr;105:482-6.

21. Milazzo L, Lai A, Pezzati L, Oreni L, Bergna A, Conti F, et al. Dynamics of the seroprevalence of SARS-CoV-2 antibodies among healthcare workers at a COVID-19 referral hospital in Milan, Italy. Occup Environ Med. 2021 Feb 4;

22. Alkurt G, Murt A, Aydin Z, Tatli O, Agaoglu NB, Irvem A, et al. Seroprevalence of coronavirus disease 2019 (COVID-19) among health care workers from three pandemic hospitals of Turkey. PLoS ONE. 2021 Mar 3;16(3):e0247865.

23. Woon YL, Lee YL, Chong YM, Ayub NA, Krishnabahawan SL, Lau JFW, et al. Serology surveillance of SARS-CoV-2 antibodies among healthcare workers in COVID-19 designated facilities in Malaysia. Lancet Reg Health West Pac. 2021 Apr;9:100123.

24. Poudel A. One in eight individuals exposed to virus until September, seroprevalence survey shows [Internet]. The Kathmandu Post. 2021 [cited 2021 Feb 26]. Available from: https://kathmandupost.com/health/2020/12/03/one-in-eight-individuals-exposed-to-virusuntil-september-seroprevalence-survey-shows

25. Malani A, Shah D, Kang G, Lobo GN, Shastri J, Mohanan M, et al. Seroprevalence of SARS-CoV-2 in slums versus non-slums in Mumbai, India. Lancet Glob Health. 2020 Nov 13;9(2):e110-1.

26. Murhekar MV, Bhatnagar T, Selvaraju S, Saravanakumar V, Thangaraj JWV, Shah N, et al. SARS-CoV-2 antibody seroprevalence in India, August-September, 2020: findings from the second nationwide household serosurvey. Lancet Glob Health. 2021 Jan 27; 
389 27. George CE, Inbaraj LR, Chandrasingh S, de Witte LP. High seroprevalence of COVID-19 infection in a large slum in South India; what does it tell us about managing a pandemic

391 and beyond? Epidemiol Infect. 2021 Feb 4;149:e39.

392 28. Himalyan News Service. 4,400 health workers infected in two months. The Himalyan

393 Times [Internet]. 2020 Nov 28 [cited 2021 Jun 4]; Available from:

394 https://thehimalayantimes.com/nepal/4400-health-workers-infected-in-two-months

395

396

397

398

399

400

401

402

403

404

405

406

407

408

409

410

411

412

413

414

415

416

417

418

419

420 


\section{Additional Information}

422

423

424

425

426

427

428

429

430

431

432

433

- Authors' Contributions

Designed the study: KRP, AB, SP, AS, PA, PG

Conducted the study: KRP, AB, SP, RB, JP, AS, PA, DA, PJP, GSS, KP, NT, ST

Laboratory investigations: $\mathrm{AB}$

Software and data analysis: KRP

First draft: KRP

Review and final draft: KRP, AB, SP, RB, JP, AS, PA, DP, PJP, GSS, KP, NT, ST, PG

- Competing interests

None of the authors have any competing interests to declare.

- Funding

This study was funded in part by the Nepal Health Research Council.

\section{Acknowledgements}

We would like to acknowledge and thank all the study participants and participating hospitals. Kamal Khadka, Kedar Prasad Century, June Kakshapati, Sharad H. Gajuryal , Dipendra Raushan, Bijesh Raj Ghimire, Basanta Maharjan, Sangeeta Mishra, Shail Rupakheti, Uma Shrestha, Polina Paudyal, Bibechan Thapa, Kailash Ghimire, Hari Prakash Oli, Yashodhara Silwal, Rukmani Khadka Basnet, Manika Maharjan, Subhash Kafle, Rajati Sharma, Jwala Dhakal, Pramisha Poudel, Junu Karki, and Manisha Neupane provided crucial support at their respective sites. Shreya Pokharel, Kabita Mahar, Dipa Shahi, Sabina Tripathi, Renuka Bhattarai helped collect samples and administer the study questionnaire. Arun Bajracharya, Rushila Chitrakar at the Department of Biochemistry, Tribhuvan University Teaching Hospital processed blood samples and performed the lab tests. Staff at Nepal Health Research Council provided crucial administrative and logistic support. 


\section{Supplementary Files}

This is a list of supplementary files associated with this preprint. Click to download.

- SEVIDKaVW1SuppAppendix.pdf 